\title{
Datenrevolution und Gesundheitswesen
}

\section{Thomas D. Szucs}

Prof. Dr. med., Verwaltungsratspräsident der Helsana-Gruppe

Korrespondenz:

Prof. Dr. med. Thomas D. Szucs, MPH, MBA, LLM Director

European Center of Pharmaceutical Medicine/ Institute of Pharmaceutical Medicine

University of Basel

Klingelbergstrasse 61

CH-4056 Basel

thomas.szucs[at]unibas.ch
Die Medizin verändert sich rasend schnell. Es handelt sich um einen Prozess der kreativen Destruktion. Die alte Medizin wird abgelöst durch eine neue Medizin, - und wir stehen mittendrin im Veränderungsprozess. Er ist eng verknüpft mit der Konvergenz von Technologien, mit Daten in der Hauptrolle. Daten sind auch in vielen anderen Bereichen der Gesellschaft von entscheidender und steigender Bedeutung. Der folgende Text beleuchtet die Datenrevolution in Medizin und Gesellschaft und fragt nach den Auswirkungen der Entwicklung auf einen innovativen Krankenversicherer.

\section{Datenflut führt zu Konvergenz der Wissenschaftsbereiche}

Das Wort Daten kommt aus dem Lateinischen (dare = geben). Daten sind etwas, das gegeben ist. In der Mathematik braucht es Daten zur Lösung von Aufgaben. Daten brauchen wir auch, um Sachverhalte darzustellen. Dabei wächst der Datenberg laufend. Viele Wissenschaftsbereiche häufen immer grössere Mengen von Daten an. Damit kommt es zu einer Konvergenz der Wissensbereiche: Insbesondere Computertechnologie, Informatik, Genomik und Bilddiagnostik rücken immer näher zusammen. In dieser Konvergenz liegt der Schlüssel für die ganz grossen, digitalen Fortschritte der Medizin. Wir haben nicht nur PC und Internet, sondern immer mehr digitale Hilfsmittel, die in der Medizin eine entscheidende Rolle spielen. Und überall werden Daten erzeugt.

Diese Daten stammen zum Teil aus dem menschlichen Körper und finden über Diagnosen und Therapien von Ärzten und Spitälern bzw. via die entsprechenden Leistungsabrechnungen auch den Weg zu den Krankenversicherern. Das Data Warehouse von Helsana arbeitet produktiv mit einer Datenmenge von rund 10 Terabyte, noch einmal so viele Daten werden in der Entwicklung eingesetzt. Das ist viel. Im Vergleich mit einem durchschnittlichen Spital aber bescheiden, wo die durchschnittliche Datenmenge 665 Terabyte beträgt, also 66-mal mehr als bei Helsana.

\section{«Big Data» - Basis für Erkenntnisgewinnung}

Im Zusammenhang mit der Bewirtschaftung der Daten spricht man von «Big Data». Dabei geht es um das zentrale Konzept, wie wir mit den Daten umgehen. Zur Analyse von Daten brauchen wir Algorithmen. Algorithmen sind nichts anderes als Rechen- anleitungen und werden täglich gebraucht. Beispiele, wie diese «Big Data Analysis» für Wissensgewinn und innovative Lösungen genutzt werden kann, gibt es immer mehr. Zum Beispiel wird der Wert von Optionen an der Börse mit Algorithmen berechnet. Es braucht einen Algorithmus, wenn wir vor mehreren Lifttüren stehen und in den 32. Stock eines Gebäudes fahren wollen. Der Algorithmus berechnet die optimale Variante, damit sich für uns schnell eine Lifttüre öffnet und gleichzeitig nicht unnötig Energie verbraucht wird. Kreditkartenfirmen können durch Analyse des Abzahlungsmusters mit einer Wahrscheinlichkeit von 95 Prozent sagen, ob ein Paar in zwei Jahren noch verheiratet ist. Die britische Firma Epagogix kann aufgrund von Datenanalysen voraussagen, welche Drehbücher ein Blockbuster werden. Und Google weiss aufgrund von Suchanfragen und Mailverkehr ebenso gut, wo die Grippe grassiert, wie das Bundesamt für Gesundheit, das sich auf die Meldungen der Ärzte stützt.

\section{Google weiss [...] ebenso gut, wo die Grippe grassiert, wie das Bun- desamt für Gesundheit.}

\section{Von «Big Data» zu «Smart Data»}

Die Bedeutung der Datenanalyse kreiert einen neuen Job, den Datenwissenschaftler. Er kanalisiert die Daten, treibt die Analyse voran. Das muss nicht immer intern geschehen. Es gibt Firmen, die ihre Daten ins Netz stellen und mit Fragen an die breite Masse der Nutzer verbinden. Wer die beste Antwort liefert, bekommt einen Preis. Diese Auslagerung von Aufgaben an eine Gruppe freiwilliger User heisst «crowdsourcing» und erfreut sich nicht nur reger Nutzung von Firmen, sondern von Wissenschaft oder Medizin. Das Pharmaunternehmen Merck hat die Methode zum Beispiel schon benutzt, um herauszufinden, ob ein Molekül sicher ist. Und die amerikanische Spitalkette Heritage wollte von der Wissenschaftscrowd im Netz wissen, wie wahrscheinlich es ist, dass jemand rehospitalisiert wird. Für Helsana ist klar: Die Auswertung von Daten muss auch uns interessieren. «Big Data» allein genügt aber nicht. Es braucht «Smart Data». 


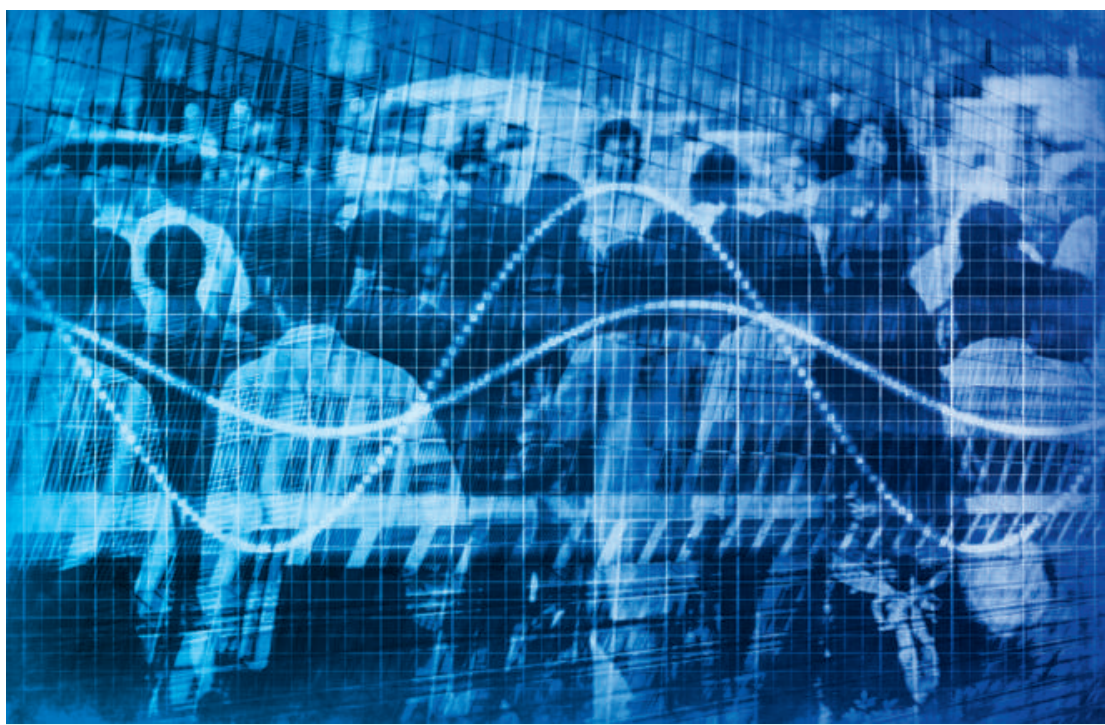

Big Data: Dank moderner Informationstechnologien lassen sich enorme Datenmengen sammeln und verarbeiten - auch in der Medizin.

\section{Interaktiver
Artikel}

Wollen Sie diesen Artikel kommentieren? Nutzen Sie dafür die Kommentarfunktion in der OnlineVersion oder sehen Sie nach, was Ihre Kolleginnen und Kollegen bereits geschrieben haben: www.saez.ch/ aktuelle-ausgabe/ interaktive-beitraege/

\section{Datenanalyse auch in der Krebsmedizin relevant}

Auch wer heute eine gute Krebsmedizin machen will, ist auf grosse Datenmengen angewiesen. Für Tumoranalysen müssen wir die Genetik des Tumors verstehen. Bestehende Technologien helfen da nicht weiter, und die neuen Verfahren sind sehr datenintensiv. Die Sequenzierung eines Tumors braucht gute Rechnerleistung. Doch es reicht nicht mehr, eine einzige Biopsie zu machen. Denn der Tumor entwickelt sich weiter. Er reagiert zum Beispiel auf Medikamente, setzt sich zur Wehr. So kommt etwas Neues zum Tragen, das unter dem Begriff «fresh biopsy» zusammengefasst wird. Das heisst nichts anderes, als dass man immer wieder neue Biopsien vom Tumor ziehen muss. Oder dass man bei einer Leukämie regelmässig das Blut analysiert. Für die Krankenversicherer bedeutet der medizinische Fortschritt also auch höhere Leistungskosten. Nicht nur eine Biopsie muss bezahlt werden, sondern mehrere.

\section{Datenaustausch unter Patienten reduziert die Hospitalisationsrate}

$\mathrm{Zu}$ guter Letzt haben auch die neuen technischen Möglichkeiten für Patienten, sich untereinander zu organisieren, soziale Netzwerke zu bilden und sich auszutauschen, einen massgeblichen Einfluss auf das Gesundheitswesen. Zum Beispiel reduziert sich durch die schiere Tatsache, dass sich die Patienten vernetzen, die Notwendigkeit von Hospitalisationen. So ist bekannt, dass zum Beispiel Epileptiker, die vernetzt sind, 18 Prozent weniger Notfallaufnahmen generieren. Und wir wissen, dass bei HIV-Patienten, die sozial zusammenarbeiten, risikobehaftetes Verhalten um 41 Prozent seltener vorkommt.

\section{Mit Datenanalyse eine Nasenlänge voraus}

Für Helsana muss die Nutzung der Datenanalyse zunächst einmal im Einklang mit der Unternehmensstrategie stehen. Wir wollen Vorreiter sowie gute Netzwerker, Begleiter und Lotsen im Gesundheitssystem sein. «Smart Data» kann uns helfen zu verstehen, was die Kunden wollen. Die Datenanalyse liefert jedoch auch Hinweise auf die künftige Kostenentwicklung. Auch helfen uns Daten zum besseren Verständnis,

\section{Durch die schiere Tatsache, dass}

\section{sich die Patienten vernetzen,}

\section{reduziert sich die Notwendigkeit von Hospitalisationen.}

welches die sicheren Medikamente sind und welche medizinische Massnahmen tatsächlich etwas bringen. Analytische Fähigkeiten stellen einen Wettbewerbsvorteil dar. Wettbewerber, die analytisch vorgehen, sind besser aufgestellt, verfügen über einen leistungsfähigeren Kundendienst und erkennen Herausforderungen lange vor der Konkurrenz. 